\title{
BAYESIAN IDENTIFICATION OF HOMOGENEOUS SUBGROUPS OF ACTORS IN A LOCAL AHP-MULTICRITERIA DECISION MAKING CONTEXT
}

\begin{abstract}
:
In our complex and interconnected society, many situations are characterised by the existence of a large number of individuals involved in the resolution of multicriteria problems. The two most commonly utilised methods in multi-actor decision making with AHP are the AIJ (Aggregation of Individual Judgments) and AIP (Aggregation of Individual Priorities). Both methods provide the collective priorities using a geometric mean of individual judgments (AIJ) and individual priorities (AIP). As is well known, when the group is not homogeneous, the geometric mean is not a representative indicator, it is therefore necessary to identify the existent heterogeneous patterns of behaviour. Following a Bayesian approach to the treatment of the log-linear model considered for the stochastic AHP, this work establishes in a local context a stochastic search procedure that allows the determination of the number and composition of the subgroups according to a maximum inconsistency threshold which is common to the decision makers. The proposed methodology is illustrated by a real-life example.
\end{abstract}

KEY WORDS: AHP, Homogeneous decision makers, Bayesian approach, Local context. 